\title{
EL GÉNERO HYPSELODESMUS \\ (DIPLOPODA: POLYDESMIDA: CHELODESMIDAE): DISCUSIÓN DE SU ESTATUS Y DESCRIPCIÓN DE UNA ESPECIE NUEVA*
}

\author{
Carlos Suriel
}

Museo Nacional de Historia Natural de Santo Domingo (MNHNSD). Calle César Nicolás Penson, Plaza de la Cultura, Santo Domingo, República Dominicana. c.suriel@museohistorianatural.gov.do

\section{RESUMEN}

Se describe una especie nueva del género Hypselodesmus y se ofrece registro de nuevas localidades para Hypselodesmus bicolor Loomis, 1941 e Hypselodesmus assoi Suriel, 2009. Las piezas gonopodales de $H$. bicolor separadas del cuerpo son ilustradas por primera vez y se incluye la hembra en una nueva descripción de la especie. Se ofrece nueva descripción para $H$. assoi. Se discute el estatus del género Hypselodesmus Loomis, 1941, endémico de La Hispaniola, proponiéndose nuevas diagnosis y descripción.

Palabras clave: Hypselodesmus, La Hispaniola, diagnosis, descripción, especie nueva, localidades nuevas.

Title: The genus Hypselodesmus (Diplopoda: Polydesmida: Chelodesmidae): discussion of its status and description of a new species.

\section{ABSTRACT}

Anew species of the genus Hypselodesmus is described and new localities for Hypselodesmus bicolor Loomis, 1941 and Hypselodesmus assoi Suriel, 2009 are reported. Isolated gonopod parts from $H$. bicolor are illustrated for the first time, and the female is included in the redescription of this specie. New description for $H$. assoi is presented. The status of the genus Hypselodesmus Loomis, 1941, endemic from Hispaniola Island is discussed, new diagnosis and description are proposed.

Key words: Hypselodesmus, Hispaniola Island, diagnosis, description, new species, new localities.

\section{INTRODUCCIÓN}

El género Hypselodesmus fue descrito por Loomis (1941) en base al examen de cinco ejemplares, tres machos maduros y dos especímenes inmaduros de un morfo al que nombró Hypselodesmus bicolor, especie tipo. Este género, endémico de La Hispaniola, se mantuvo como monotípico por más de seis décadas, hasta la descripción de una segunda especie, Hypselodesmus assoi, por el autor de este artículo (Suriel, 2009). En esa ocasión, advertíamos la pertinencia de una nueva descripción del género, en consideración de la variabilidad que comenzábamos a conocer alrededor de un criterio fundamental y unitario, el diseño y la estructura particulares de los gonopodos: "largos, alcanzando el par posterior de patas de S6...con fémur (o acropodito, nota a posteriori), acintado, flexionado en forma de "s" abrazando el proceso prefemoral, con postfémur igualmente acintado y curvado hacia fuera, características inconfundibles con especie

\footnotetext{
*Las expediciones que permitieron reunir el material necesario para este trabajo se realizaron en el marco del proyecto $2009-102$ financiado por FONDOCYT, del Ministerio de Educación Superior, Ciencia y Tecnología (MESCYT), bajo la dirección del Instituto de Investigaciones Botánicas y Zoológicas de la UASD y la responsabilidad compartida del Museo Nacional de Historia Natural.
} 
alguna en la fauna de diplópodos de La Hispaniola" (Suriel, 2009, p.4). Este rasgo distintivo fue propuesto para Hypselodesmus en la división de la familia Chelodesmidae en seis géneros válidos, pudiendo ser resumido, a partir de una clave dicotómica publicada, de la siguiente manera: gonopodos que sobrepasan el borde anterior del esternito del séptimo segmento...con acropodito no birramoso...plegado en su porción distal (Pérez-Asso, 2010, p.11).

Aunque ajustada muy bien a esta razón de discriminación genérica, la especie nueva, $H$. assoi, se describía presentando variaciones con respecto a la especie tipo, tales como la mayor longitud del prefémur, un postfémur con extremo no bifurcado, agudo, acropodito abrazando solo parcialmente al proceso prefemoral, destacándose también diferencias obvias con respecto a esta última estructura; en adición, otros rasgos de $H$. assoi se describieron como diferentes, entre ellos: segmentos con igual patrón de coloración, sin diferencia entre segmentos poríferos y no poríferos en el ángulo posterior del paraterguito, el que tan solo presenta un tono más claro, ambarino, no el color blanco propio de $H$. bicolor, y diente en ángulo anterior de paraterguito sin regularidad (Suriel, 2009).

El descubrimiento reciente de una tercera especie, la que se describe en el presente artículo, con muy obvias diferencias respecto a las dos ya conocidas, pero con características inconfundibles de Hypselodesmus, ofrece la oportunidad para una nueva descripción del género que tome como eje el criterio unitario ya destacado e incluya la variabilidad comprendida por las tres especies. Se aprovecha el espacio para actualizar la descripción de $H$. bicolor con la inédita ilustración de las piezas gonopodales separadas y el examen de nuevas poblaciones descubiertas; también se incluye la hembra en esta descripción, la que no fue contemplada por Loomis en su trabajo por falta de ejemplares. El examen de una amplia serie de topotipos y otros especímenes colectados de $H$. assoi se utiliza para una nueva descripción de esta especie.

\section{OBJETIVOS}

- Describir una especie nueva del género Hypselodesmus.

- Presentar una nueva descripción de H. assoi Suriel, 2009.

- Hacer una nueva descripción de H. bicolor Loomis, 1941, incluyendo la hembra de la especie.

- Examinar y actualizar la situación del género Hypselodesmus Loomis, 1941, con nuevas diagnosis y descripción.

\section{MATERIALES Y MÉTODOS}

Todos los especímenes examinados se preservaron en alcohol etílico al 70\%: H. bicolor, cuatro topotipos, 29 ejemplares de Loma Barbacoa (nuevo registro: 13 machos, 16 hembras) y tres ejemplares de Reserva Científica Ébano Verde (dos machos y una hembra); H. assoi, el holotipo, cinco paratipos (tres machos, dos hembras), 17 topotipos (11 machos y 6 hembras) y siete ejemplares de otras localidades; Hypselodesmus sp. nov., 13 ejemplares, el asignado como tipo portanombre (holotipo) y 12 asignados como paratipos (ocho machos y cuatro hembras). Se usó un microscopio estereoscópico MEIJI TECHNO modelo EMZ-5TR para las observaciones. Todos los dibujos fueron realizados por el autor, utilizando una cámara lúcida K400 en un microscopio estereoscópico Motic, serie K, S/N S907147. Las ilustraciones realizadas fueron luego escaneadas y limpiadas con Photoshop versión CS5 para su corrección. Las fotos de partes del cuerpo se tomaron usando una cámara Nikon, modelo Coolpix 4500, instalada en un microscopio estereoscópico marca Leica, modelo MZ7.5, procesándose las imágenes con el programa Helicon Focus, versión 4.2.7x64; fotos de cuerpo entero con cámara Nikon D300, 
con micronikor $105 \mathrm{~mm}$. Las mediciones se hicieron con una miniescala de Bio Quip Products, Inc. modelo "Métrica" con un rango de $5 \mathrm{~mm}$ y divisiones de $0.1 \mathrm{~mm}$, así como una regla milimetrada transparente. Se usó un GPS marca Garmin, modelo ETREX, para el registro de las coordenadas geográficas y la altitud de las localidades.

La nomenclatura taxonómica sigue a Hoffman (1979), las medidas de longitud y anchura del cuerpo de los diplópodos se tomaron de acuerdo a Pérez-Asso (1996), el valor ofrecido en cada caso corresponde a la media, indicándose entre paréntesis el rango de variación y el número de ejemplares medidos. Abreviaturas usadas: MNHNSD=Museo Nacional de Historia Natural, Santo Domingo; SX=número del diplosegmento correspondiente (ejemplo: S7=séptimo segmento); ARPA=Colección Antonio R. Pérez-Asso.

\section{RESULTADOS}

Taxonomía. Clase Diplopoda DeBlainville In Gervais, 1844; Subclase Helminthomorpha Pocock, 1887; Superorden Merocheta Cook, 1895; Orden Polydesmida Leach, 1814; Suborden Chelodesmidea Cook, 1895; Superfamilia Chelodesmoidea Cook, 1895; Familia Chelodesmidae Cook, 1895; Subfamilia Chelodesminae Hoffman, 1979; Género Hypselodesmus Loomis, 1941 (Hoffman, 1979).

Referencias consideradas. Hypselodesmus Loomis, 1941, Bull. Mus. Comp. Zool., 88 (2): 53-55. Jeekel, 1971, Monog. Nederl. Entom. Vereng., 5: 267. Hoffman, 1979, Mus. Hist. Nat. Genéve: 154; 1999, Virg. Mus. Hist. Nat., Spec. Public., 8: 288. Pérez-Asso y Pérez-Gelabert, 2001, Bol. S.E.A. 28: 74. Perez-Gelabert, 2008, Zootaxa, 1831: 72. Suriel, 2009, Novit. Caribaea, 2: 1-6. Index to organism names (ION), http://www.organismnames.com/query.htm?q, mayo, 2012, Thomson Reuters' Zoological Record.

\section{Hypselodesmus subtilissimus sp. nov.}

Figuras 1, C-E; 2, A-F; 4, A-C

Diagnosis. Gonopodos de acropodito simple, no birramoso, acintado y arqueado, que abraza al proceso prefemoral, con postfémur largo, estrecho y plegado, de extremo notablemente filiforme curvado hacia fuera. Se diferencia de Hypselodesmus bicolor e Hypselodesmus assoi por tener su dorso conspicuamente granuloso, con los granos o tubérculos bien definidos, dispuestos en tres hileras transversales, una anterior y dos posteriores a una depresión muy marcada en el centro del terguito, no tenue como en aquellas otras dos especies, en las que los granos son muy pequeños o solo aparecen insinuados y no se definen en tres hileras. El postfémur no presenta un proceso dentiforme, como en $H$. bicolor e H. assoi, y su porción distal es marcadamente filiforme.

Diagnosis. Gonopods with a simple, single branched, ribbon-like acropodite, bent around and behind the prefemoral process. Postfemur is long, slender and folded with a remarkably filiform, outwardly curved end. Distinguished from Hypselodesmus bicolor and Hypselodesmus assoi by its conspicuously granular dorsum, with well-defined grains, arranged in three transverse rows, one anterior to and two posterior to a very marked depression in the center of the terga, which is less significant in the other two species, whose grains are very small, appearing only slightly, and not defined in three rows. Unlike $H$. bicolor and $H$. assoi, the postfemur is without dentiform process and its distal portion is notably filiform.

Descripción. Los gonopodos sobrepasan borde anterior del séptimo segmento (S7), diseño relativamente simple (Fig. 1C). Prefémur mediano en tamaño. Acropodito de una sola rama, acintado y curvado, articulado con prefémur en ángulo de casi 90 grados, más alto que el proceso prefemoral, abrazándolo, más grueso y ancho en su base, estrechándose hacia arriba, 
su postfémur es largo, igualmente acintado, sin proceso dentiforme, con extremo notablemente filiforme, largo y curvado hacia fuera (Figs. 1, C-E; 2, A-C). Proceso prefemoral fornido, más estrecho en la base, en forma de cinta gruesa en porción media, abriendo en porción superior como una lámina retorcida, con cara ventral excavada (la cara que se observa en vista posterior de gonopodos in situ) mirando hacia arriba, extremo alargado, irregular o agudo. La cara dorsal de la porción superior (la que se observa en vista anterior de gonopodos in situ) es convexa. (Figs. 1C; 2, D-E).

Tamaño mediano. Cabeza grande, con surco longitudinal pronunciado. Antenas extendidas hacia atrás regularmente sobrepasan margen anterior $\mathrm{S} 3$, no siempre alcanzan margen posterior de S4. Collum pequeño en relación al tamaño de la cabeza, elíptico, emarginado en porción media de su margen posterior, granuloso, con pequeña depresión anterocentral. Dorso ligeramente aplanado, terguitos pequeños en relación al volumen de los segmentos, más notorio en las hembras. Primeros tres segmentos de igual tamaño que el resto. Color castaño rojizo, sin diferencia entre segmentos poríferos y no poríferos, más claro en margen posterior del metaterguito y en paraterguito, más oscuro en proterguito y pleurito. Superficie dorsal con granos o tubérculos prominentes dispuestos en tres hileras transversales, una anterior y dos posteriores a una depresión central muy marcada, la última hilera se arregla junto al margen posterior del terguito o sobre este, en algunos ejemplares estas dos hileras localizadas debajo de la depresión tienden a unirse; algunos granos se extienden hasta el área del paraterguito y ocupan su margen posterior, también están presentes en el collum; número de tubérculos por terguito es variable. Ausencia de setas dorsales (Fig. 4, A-C).

Paraterguitos reducidos, con margen superior ligeramente redondeado y diente en su ángulo anterior, regularmente a partir de S2 hasta segmentos posteriores, S17 o S18. Un segundo diente presente en margen lateral del paraterguito a partir de segmentos anteriores, S2 o S3, hasta segmentos posteriores con ocurrencia variable: ausente en algunos segmentos, presente de un solo lado o apenas insinuado en uno o en ambos lados; este segundo diente suele ser más grande que el primero, pero menos agudo, se localiza en la porción media o en el tercio anterior del margen lateral, en algunos segmentos se observa ligeramente hendido y aparentando una tendencia a dividirse en dos. Un tercer diente, de ocurrencia muy variable en los segmentos, puede estar presente, regularmente agudo. El margen lateral del paraterguito de S2 suele ser irregular. Ángulo posterior del paraterguito agudo y extendido, sobretodo en segmentos no poríferos, inclinado hacia arriba, se dirige hacia fuera sobrepasando el margen lateral del cuerpo, en últimos segmentos se inclina notablemente hacia atrás y se hace más largo. Callo porífero prominente, reduciéndose apreciablemente en últimos tres o dos segmentos (Fig. 4, A-C). Fórmula porífera normal (5, 7, 9-10, 12-13, 15-19), los poros abren dorso-lateralmente. Epiprocto alargado, doblado hacia abajo en su extremo (Fig. 4C).

Esternitos castaño claro. Adaptación pregonopodal de los machos consistiendo en prominencia cónica sobre coxa de segundo par de patas. Patas gruesas, las pregonopodales profusamente pilosas, tercer y sexto podómeros más largos y casi del mismo tamaño. Hipoprocto redondeado, valvas anales estriadas.

Holotipo. Macho, número catalográfico MNHNSD 19. 2,271. Longitud=31.0 mm, anchura=4.1 mm. Figuras: 1C; 4, A-C.

Vista dorsal. Cabeza grande en relación al tamaño del collum. Antenas extendidas hacia atrás alcanzan S3 pero sin sobrepasar su margen posterior. Collum elíptico, emarginado en porción media del margen posterior. Color castaño rojizo, más claro en margen posterior del terguito y en paraterguito. Sin diferencia de color entre segmentos poríferos y segmentos no poríferos, proterguito y pleurito de tono más oscuro. 

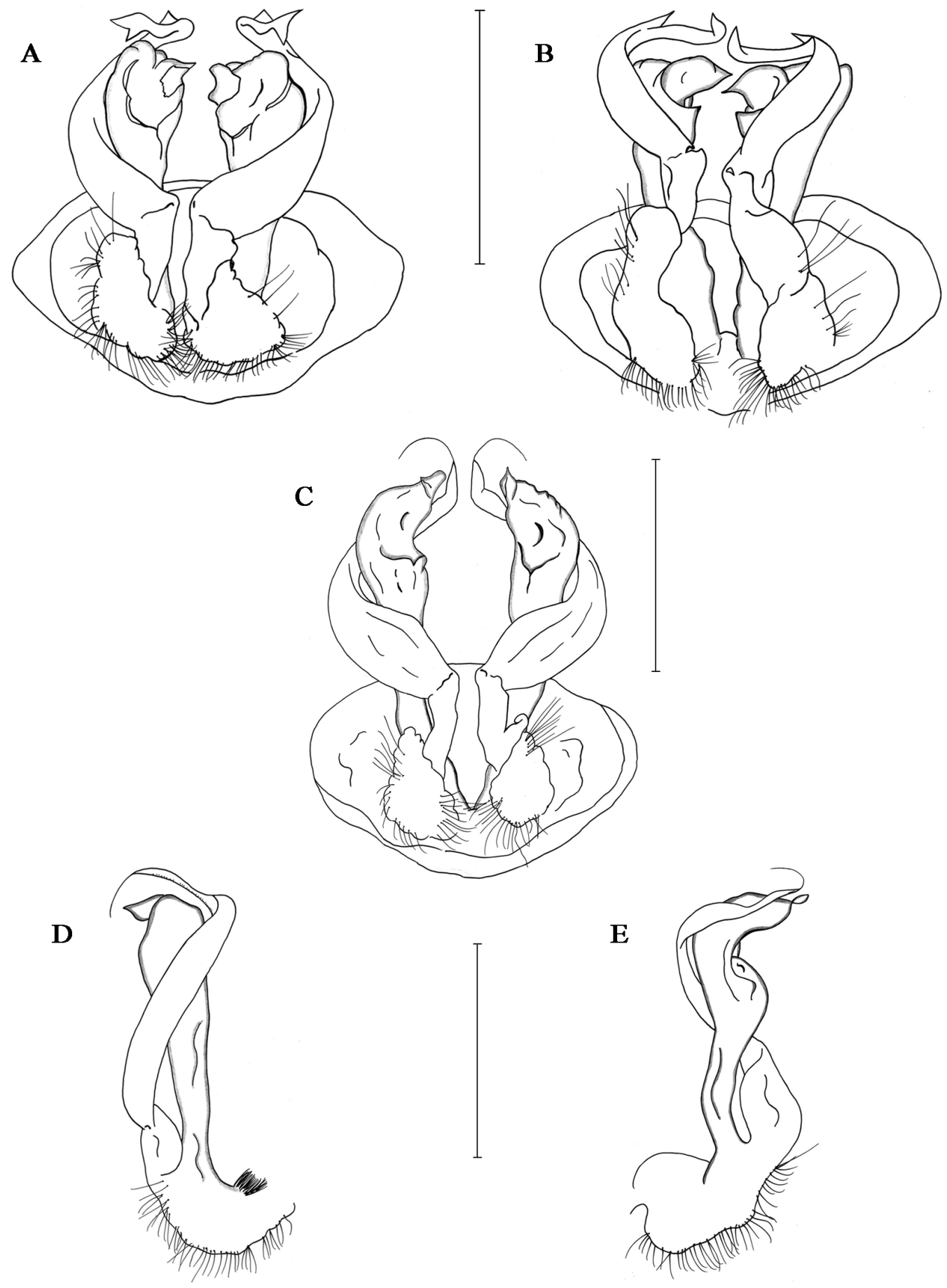

D

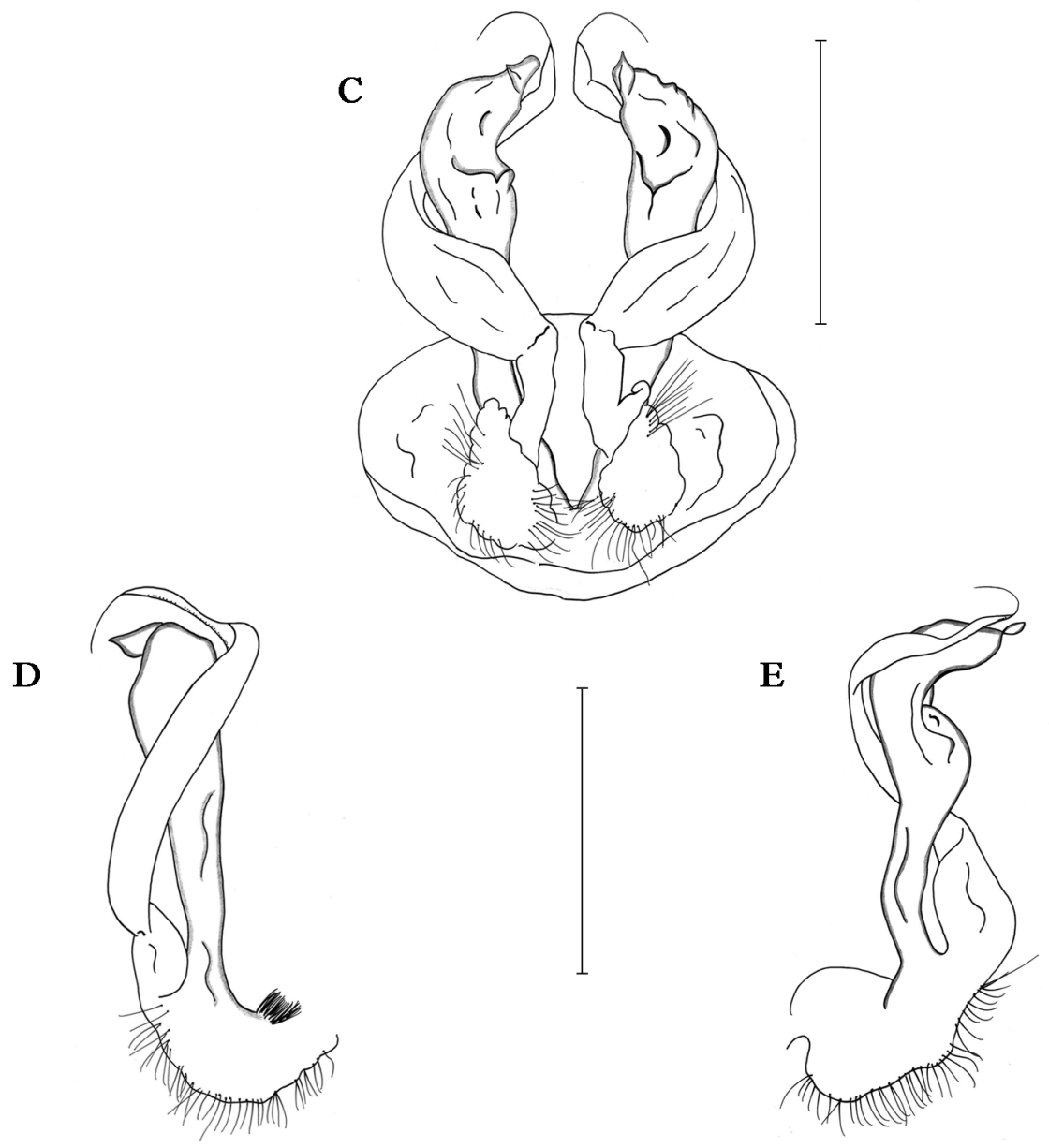

Figura 1. Gonopodos varios. A, vista posterior de gonopodos in situ de Hypselodesmus bicolor (topotipo). B, vista posterior de gonopodos in situ de H. assoi (paratipo). C, vista posterior de gonopodos in situ de H. subtilissimus sp. nov. (holotipo, MNHNSD 19.2,271). D-E, gonopodo derecho desprendido de H. subtilissimus sp. nov. (paratipo MNHNSD $19.2,276$ ): D, vista posterolateral; E, vista antero-lateral. Escala $=1 \mathrm{~mm}$. Bordes del proceso prefemoral sombreados para diferenciar. 
A

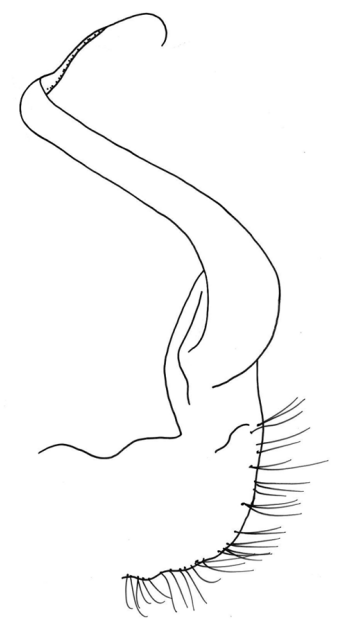

C

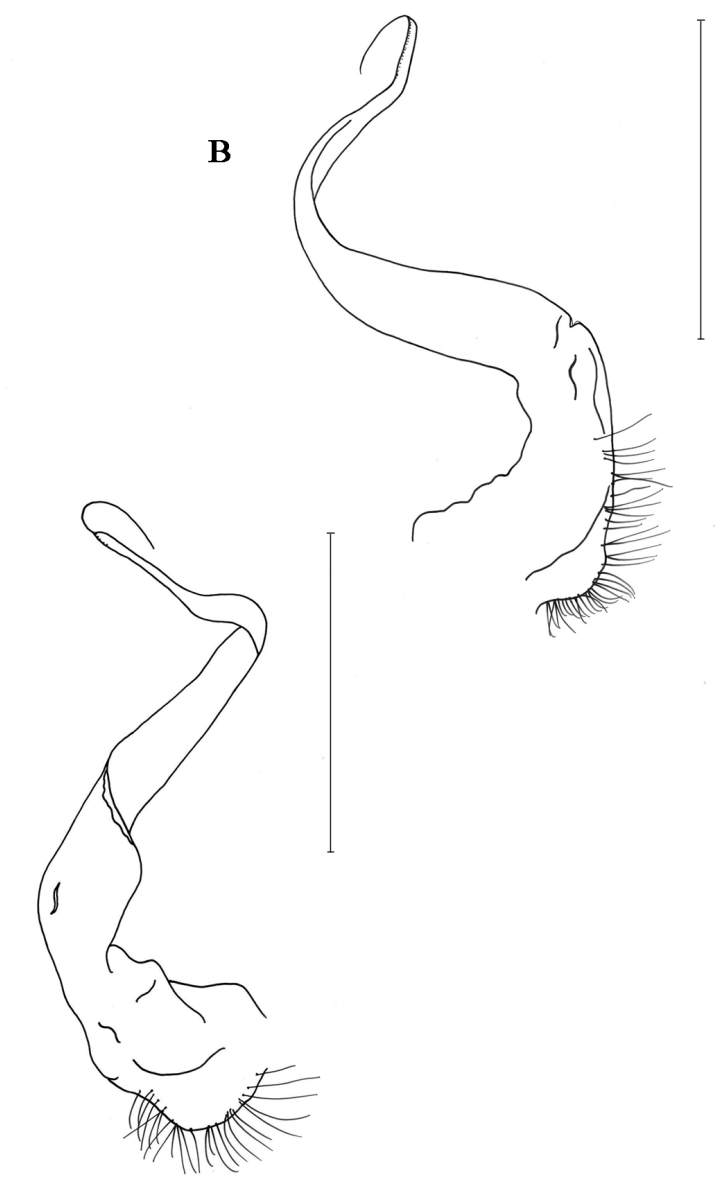

D

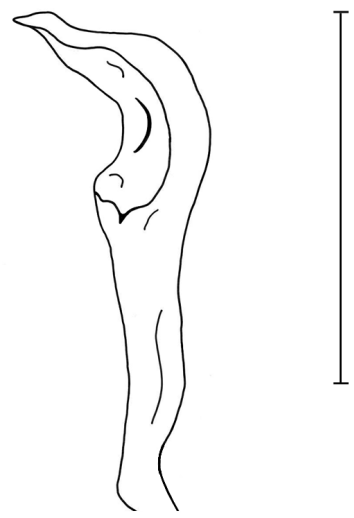

$\mathbf{E}$

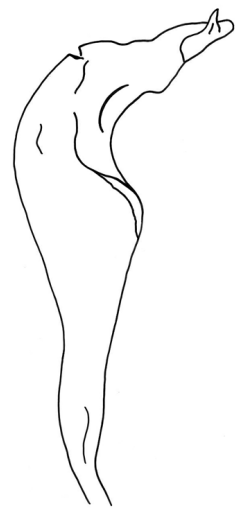

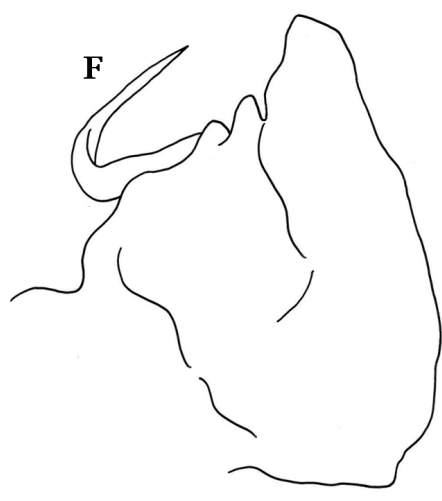

Figura 2. Piezas gonopodales separadas de Hypselodesmus subtilissimus sp. nov. (gonopodo izquierdo de paratipo MNHNSD J 19.2,276). A-C, acropodito: A, vista postero-lateral; B, vista postero-lateral, pieza girada ligeramente hacia la izquierda; C, vista antero-lateral. D-E, proceso prefemoral: D, vista postero-lateral; E, vista posterior. F, coxa con cánula. Escala=1mm. 
Cuerpo ligeramente aplanado, con terguitos relativamente pequeños. Presencia de granos o tubérculos dispuestos en tres hileras transversales, una anterior y dos posteriores a una depresión central marcada, igualmente transversal. En los segmentos posteriores los granos o tubérculos se levantan notoriamente en forma de papilas y los de las dos hileras posteriores tienden a juntarse, en algunos segmentos se arreglan sobre el borde posterior del terguito. Algunos de los granos ocupan el área del paraterguito. Ausencia de setas. Margen anterior de paraterguitos ligeramente redondeado. Margen lateral de S2 irregular. Diente agudo en ángulo anterior de paraterguito (S2-S18); un segundo diente, menos agudo, en el margen lateral: S2 insinuado, S3-S8 presente en ambos lados, S9-S10 presente solo del lado derecho, S11 presente en ambos lados, S12-S13 presente del lado derecho e insinuado del lado izquierdo, S14-S15 presente en ambos lados, S16 presente del lado derecho e insinuado del lado izquierdo, S17-S18 insinuado en ambos lados. Un tercer diente en lado derecho de S5. La depresión central del metaterguito es muy marcada a partir de S7, haciéndose más obvio el levantamiento del paraterguito, sobretodo de su ángulo posterior, el que se dirige hacia arriba. Ángulo posterior de paraterguito agudo, alargado a partir de S3, extendido lateralmente en segmentos anteriores, hacia atrás en segmentos posteriores, sobretodo en S17-S19. Fórmula porífera normal, callo porífero conspicuo, poros abren lateralmente y hacia arriba. Epiprocto extendido e inclinado hacia abajo.

Vista ventral. Surco longitudinal en la cabeza. Patas gruesas, las anteriores profusamente pilosas, con tercer y sexto podómeros más largos, adaptación especial en forma de prominencia cónica sobre la base de las patas del segundo par. Hipoprocto redondeado, valvas estriadas. Esternitos castaño claro, los anteriores a S7 más pilosos.

Gonopodos. Alcanzan S6, midiendo aproximadamente $1.5 \mathrm{~mm}$ desde la porción media del prefémur hasta el punto más elevado del postfémur, donde su extremo filiforme se curva. Acropodito curvado, se articula con prefémur en ángulo de casi $90^{\circ}$, acintado, notablemente ancho en su base, estrechándose hacia arriba, rodea al proceso prefemoral, abrazándolo; postfémur sin ningún proceso dentiforme, terminando en un extremo filiforme curvo, doblado hacia fuera. Prefémur mediano en tamaño. Proceso prefemoral estrecho en la base, ascendiendo en forma de una banda gruesa y abriendo como lámina retorcida en su porción superior, con cara gruesa, excavada y de extremo irregular, mirando hacia arriba.

Variabilidad y regularidades morfológicas. Machos: longitud $=30.7 \mathrm{~mm}(29.0-32.0 ; \mathrm{n}=9)$, anchura $=3.96 \mathrm{~mm}(3.8-4.1 ; \mathrm{n}=9)$. Hembras: longitud $=33.1 \mathrm{~mm}(32.0-34.0 ; \mathrm{n}=5)$, anchura $=$ $4.28 \mathrm{~mm}(4.1-4.5 ; \mathrm{n}=5)$. Una hembra, 19. 2,283, solo fue considerada para las mediciones del cuerpo debido a la falta de antenas y varios pares de patas.

Gonopodos. Los extremos filiformes, el de cada acropodito, pueden hacer contacto en el punto donde curvan hacia fuera $(ð 19.2,272)$ o entrecruzarse $(\lesssim 19.2,270)$. El proceso prefemoral puede ser notablemente estrecho en su origen $(\hat{\jmath} 19.2,273)$. No se observaron otras variaciones gonopodales de consideración.

Dimorfismo. Las hembras presentan cuerpo ligeramente más grande (hasta $34 \mathrm{~mm}$ de longitud y $4.5 \mathrm{~mm}$ de anchura), al tener los segmentos más voluminosos se aprecian sus terguitos levemente de menor tamaño que en los machos. Las patas de las hembras son más finas y menos pilosas y el tercer podómero es más largo que en los machos; esternitos en base de las patas con menos setas.

En todos los ejemplares examinados la cabeza se aprecia grande en relación al tamaño del collum, el cual la sobrepasa un poco lateralmente, pero no la cubre por delante suficientemente; el patrón de color también presenta mucha regularidad (excepto $\lesssim$ 19.2,277, de un castaño mucho 
más claro que los demás, amarillento), al igual que la fórmula porífera (siempre normal) y el callo porífero. Las antenas extendidas hacia atrás regularmente alcanzan S3, en algunos casos

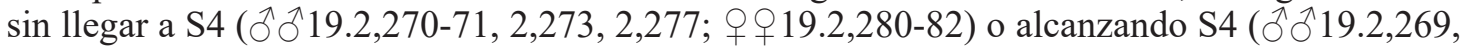
2,272, 2,275-76, 2,278; $919.2,274)$. La depresión transversal en el centro del metaterguito se hace muy notoria a partir de S5. Los granos son conspicuos, las dos hileras posteriores se levantan como papilas puntiagudas y tienden a juntarse en los últimos segmentos. El diente en ángulo anterior de paraterguito aparece regularmente, S2-S18 de todos los ejemplares examinados, no así el segundo diente, más abajo, el que presenta una expresividad muy variable en cuanto a su ocurrencia, apareciendo desde S2 o S3 pero pudiendo estar solo insinuado de ambos lados o de uno, presente en un solo lado, presente en ambos lados o ausente totalmente en algunos segmentos; el tercer diente es de ocurrencia más variable: đ̋19.2,269 en S14; đ̋ 19.2,271 en S5; $\widehat{~} 19.2,272$ en S5 solo del lado derecho; + 19. 2,274 solo insinuado del lado derecho de S2 y \$19.2,282 lado derecho de S14; puede estar totalmente ausente en algunos ejemplares. Ángulo posterior de paraterguito regularmente agudo y extendido a partir de S3 o de S4, inclinado lateralmente, poco inclinado hacia atrás excepto en los últimos segmentos, sobre todo si se le compara con las otras especies del género. Los esternitos son de tono más claro que el dorso, excepto en +19 . 2,274 donde se presentan castaño oscuro. Hipoprocto y valvas anales no presentan variaciones de consideración, a no ser el grado de expresividad de las estrías en las valvas.

Etimología. El epíteto específico subtilissimus, superlativo de subtilis = fino, alude al muy fino extremo del postfémur de esta especie, mucho más que en las otras dos especies conocidas, notablemente filiforme.

Comentarios. El extremo filiforme del acropodito es el rasgo más distintivo de esta especie, no obstante, su dorso conspicuamente granuloso, con los granos o tubérculos organizados claramente en tres hileras y la depresión central del metaterguito bien marcada constituyen otras características diferenciadoras. A simple vista, el cuerpo de $H$. subtilissimus sp. nov. luce menos aplanado que los de $H$. bicolor e $H$. assoi, y la presencia de los granos o tubérculos levantados confiere un aspecto particular a la superficie dorsal.

La menor inclinación hacia atrás del ángulo posterior del paraterguito es otro rasgo fácilmente apreciable en $H$. subtilissimus sp. nov. Los ejemplares de esta especie nueva de quelodésmido aparecieron muy asociados con una población de $H$. bicolor (población de nuevo registro), enterrados y entre restos de vegetación en descomposición de un manaclar a 1,227 msnm. Se diferencia a simple vista de H. bicolor por la uniformidad de su coloración dorsal, no teniendo diferencia entre segmentos; así mismo, otro rasgo muy distintivo y de fácil apreciación es la superficie áspera de su dorso por la presencia abundante de los granos o tubérculos en sus terguitos. Otros milpiés que se encontraron asociados son dos morfos del género Prostemmiulus (familia Stemmiulidae) y otro de la familia Rhinocricidae.

Tipos y distribución geográfica. Al momento, Hypselodesmus subtilissimus sp. nov. solo se conoce de la localidad tipo: un manaclar de bosque nublado a 1,227 msnm en la loma Barbacoa, provincia Peravia, subiendo desde La Taguía, provincia San Cristóbal, aproximadamente tres horas en mulos, siendo sus coordenadas 358585 E, 2041750 N. Holotipo MNHNSD Љ19. 2,271. Paratipos: MNHNSD §ð19.2,269-70, 19.2,272-73, 19.2,275-78; 우19.2,274, 2,28082. Colectores: "Naná", "Chelín”, "Santico" (monteros que sirvieron de guías) y Carlos Suriel 18-III-2011 y 01-X-2011. Todos los tipos han sido depositados en la colección de diplópodos del MNHNSD, excepto los paratipos $\overbrace{}^{\top} 2,275$ y $\$ 2,274$, los cuales pasan en intercambio a la colección ARPA. 


\section{Hypselodesmus assoi}

Figuras 1B; 4, D-F

Hypselodesmus assoi Suriel, 2009. Novit. Caribaea 2: 1-6, figs. 1-3, 5-7. Index to organism names (ION), http://www.organismnames.com/query.htm?q., citado en mayo, 2012, Thomson Reuters' Zoological Record (145).

Nueva descripción. Gonopodos grandes, sobrepasan el margen anterior del esternito del séptimo segmento. Prefémur largo. Acropodito acintado, curvado, más alto que el proceso prefemoral, al que abraza solo parcialmente, descansando sobre depresión acanalada longitudinal de éste, cubriendo parte de su margen interno; postfémur angosto, acintado, con proceso dentiforme, plegado y curvado hacia fuera, con extremo agudo, ambos pueden tocarse o entrecruzarse en algunos casos (Fig. 1B). Proceso prefemoral fornido, estrecho en la base, porción media como cinta gruesa, la porción superior abre como lámina retorcida, con cara ventral excavada amplia, mirando lateralmente y hacia arriba, extremo extendido, lanceolado y agudo, dirigido lateralmente o retorcido hacia arriba; presenta una depresión acanalada, longitudinal y oblicua, donde descansa el acropodito. La cara dorsal de la porción superior es ligeramente cóncava. Dorso color castaño, un poco más claro en porción central de metaterguito, principalmente en el margen posterior. Sin diferencia de color entre segmentos poríferos y no poríferos, ángulo posterior de paraterguito ligeramente más claro, proterguito y pleurito más oscuros. Collum subelíptico, pequeña depresión anterocentral con setas a los lados, emarginado en el centro de su margen posterior. Antenas largas, extendidas hacia atrás sobrepasan margen anterior del cuarto segmento, pueden llegar al margen posterior del quinto segmento. Primeros tres segmentos ligeramente más anchos que el resto. Segmentos ligeramente convexos en el centro, sobretodo en segmentos anteriores, con una muy tenue depresión transversal en metaterguito, no siempre observable en segmentos anteriores; pequeños granos, frecuentemente sutiles, dispersos en el terguito, en segmentos posteriores tienden a organizarse en una hilera debajo de la depresión central. Setas ausentes. Paraterguito con margen anterior redondeado, ligeramente levantado, diente en su ángulo anterior de expresividad muy variable a partir del segundo segmento, pudiendo faltar en un lado del segmento o estar tan solo insinuado, en ocasiones tan solo en S2-S4, regularmente ausente en segmentos posteriores; área de paraterguito más reducida en las hembras, su cuerpo es notoriamente menos aplanado. Ángulo posterior de paraterguito agudo y extendido hacia fuera, progresivamente inclinado hacia atrás, sobretodo en los machos y en segmentos posteriores, reduciéndose su área en los últimos segmentos; se inclina hacia arriba a partir de los segmentos medios o desde segmentos anteriores (con frecuencia desde S5) con poro que abre lateralmente y hacia arriba en fórmula normal $(5,7,9-10,12-13,15-19)$, callo porífero alargado y ovalado. Epiprocto alargado, con extremo flexionado hacia abajo (Fig. 4, D-F). Surco bien marcado en el vertex de la cabeza, con setas a los lados. Adaptación pregonopodal en segundo par de patas, consistiendo en sendas prominencias cónicas alargadas sobre las coxas. Patas largas y finas, pilosas, con el tercer y sexto podómeros más largos. Esternitos de tono más claro que el dorso. Hipoprocto más ancho que largo, valvas anales estriadas.

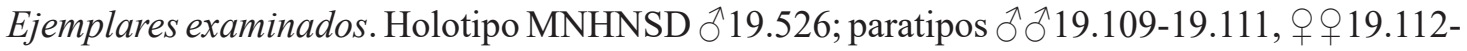
19.113. Localidad tipo: Sabana Vieja, provincia San Juan, Parque Nacional José del Carmen Ramírez (Cordillera Central), República Dominicana, 1904'45' N, 071 $11^{\prime} 52^{\prime \prime} \mathrm{W}, 1,944$ msnm, entre pajones e hierbas y bajo corteza en un pinar. Colectores: Rosa Rodríguez, Gabriel de los Santos, Alexander Sánchez-Ruiz, Elvi de los Santos, Miguel Ángel Landestoy y Carlos

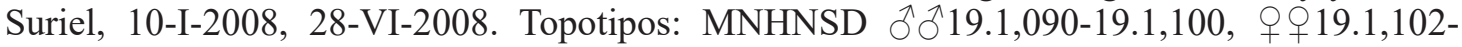
19.1,103, 19.1,106-1,108, 19.1,112. Colectores: Gabriel de los Santos, Robert Ortiz y Carlos Suriel, 23-XI-2009. MNHNSD స̂.19.1,000, Loma El Atrecho, Parque Nacional José del Carmen Ramírez (Cordillera Central), provincia San Juan, 1902'51.7' N-71¹3'04.4' 'W, 1,702 msnm; 
G. de los Santos, R. Ortiz y C. Suriel, 22-XI-2009. MNHNSD 19.1,048-1,052 (2ðð y 3 ๆ Alto de la Rosa, Parque Nacional José del Carmen Ramírez (Cordillera Central), provincia San Juan, $19^{\circ} 02^{\prime} 21^{\prime \prime} \mathrm{N}-71^{\circ} 13^{\prime} 20.8^{\prime \prime} \mathrm{W}, 1,773 \mathrm{msnm}$; G. de los Santos, R. Ortiz y C. Suriel, 22-XI-

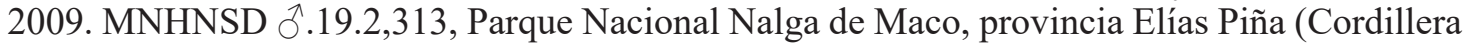
Central), $19^{\circ} 12^{\prime} 15.8^{\prime \prime} \mathrm{N}-71^{\circ} 28^{\prime} 58.4^{\prime \prime} \mathrm{W}, 1,965$ msnm, Katihuska Rodríguez, 7-8-IV-2012.

Registro de nuevas localidades. Loma El Atrecho, Parque Nacional José del Carmen Ramírez (Cordillera Central), provincia San Juan, 1902'51.7' N-71'13'04.4' 'W, 1,702 msnm, 22-XI2009. Alto de la Rosa, Parque Nacional José del Carmen Ramírez (Cordillera Central), provincia San Juan, $19^{\circ} 02^{\prime} 21^{\prime \prime} \mathrm{N}-71^{\circ} 13^{\prime} 20.8^{\prime \prime} \mathrm{W}, 1,773 \mathrm{msnm}, 22-\mathrm{XI}-2009$. Parque Nacional Nalga de

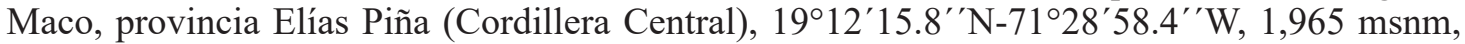
7-8-IV-2012.

Comentario. La expresividad de la depresión transversal en metaterguito y la presencia de granos en $H$. assoi es menor que en $H$. bicolor. Los granos no fueron advertidos en el holotipo y los paratipos de la descripción de la especie, el presente artículo incorpora esta característica como resultado del examen de una serie de especímenes topotipos, en algunos de los cuales son notorios y se observan con tendencia a formar una hilera debajo de la tenue depresión central.

Hypselodesmus bicolor

Figuras 1A; 3, A-F; 4, G-L

Hypselodesmus bicolor Loomis, 1941, Bull. Mus. Comp. Zool., 88 (2): 54-55, fig.19. Jeekel, 1971, Monog. Nederl. Entom. Vereng., 5: 267. Hoffman, 1979, Mus. Hist. Nat. Genéve: 154; 1999, Virg. Mus. Hist. Nat., Spec. Public., 8: 288. Pérez-Asso y Pérez-Gelabert, 2001, Bol. S.E.A. 28: 74. Pérez-Gelabert, 2008, Zootaxa, 1831: 72. Suriel, 2009, Novit. Caribaea, 2: 1-6. Index to organism names (ION), http://www.organismnames.com/query.htm?q, mayo, 2012, Thomson Reuters' Zoological Record (79).

Nueva descripción. Gonopodos alcanzan margen anterior del séptimo segmento (S7). Prefémur de tamaño mediano. Acropodito articulado con prefémur en ángulo de casi $90^{\circ}$, acintado y arqueado, abraza al proceso prefemoral; postfémur largo, plegado y curvado hacia fuera, con proceso dentiforme y extremo bifurcado (Fig. 1A) Proceso prefemoral fornido, más corto que el acropodito, parcialmente ocultado por el acropodito, estrecho en la base, porción media acintada y gruesa, con depresión acanalada longitudinal y oblicua en la que descansa el acropodito, su porción superior abre como lámina retorcida, con cara ventral excavada que mira hacia arriba y extremo irregular que se inclina lateralmente y hacia arriba, su cara dorsal es cóncava. Cuerpo mediano, el de las hembras ligeramente más grande, menos aplanado. Color castaño, más claro en el centro de margen posterior de metaterguito, proterguito y pleurito más oscuros, paraterguito o su ángulo posterior blanco en segmentos poríferos y en los tres primeros segmentos, puede ser más claro o blanquecino en algunos segmentos no poríferos, estos últimos pueden ser color castaño uniformemente, en ocasiones mucho más oscuros que los segmentos poríferos. Los tres primeros segmentos ligeramente más anchos que los demás. Collum elíptico, ligeramente emarginado en porción central de margen posterior, con pequeña depresión antero central. Margen anterior de paraterguito redondeado, con diente regularmente desde S2 hasta segmentos posteriores (S17-S18). Paraterguito ligeramente levantado, amplio, con ángulo posterior inclinado hacia arriba, con menos expresividad en las hembras. Metaterguito con depresión transversal en el centro de expresividad variable en su profundidad, frecuentemente a partir de S5, notoria o tenue, con granos o tubérculos pequeños dispersos, anteriores y posteriores a la depresión, los posteriores tienden a organizarse en una hilera junto al margen posterior del terguito; los granos pueden ocupar área de paraterguito, en ocasiones se observan los anteriores 
A

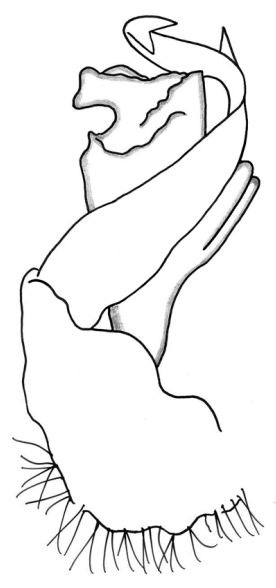

C

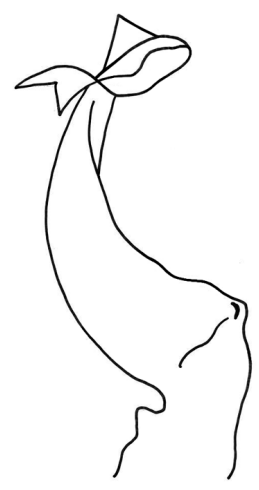

$\mathbf{E}$

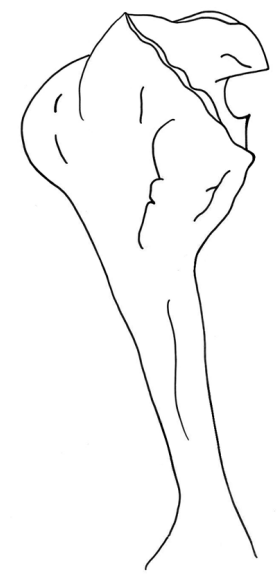

B

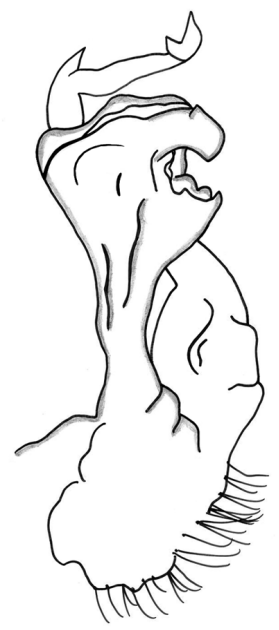

D

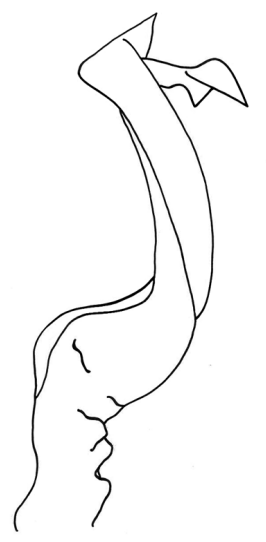

$\mathbf{F}$

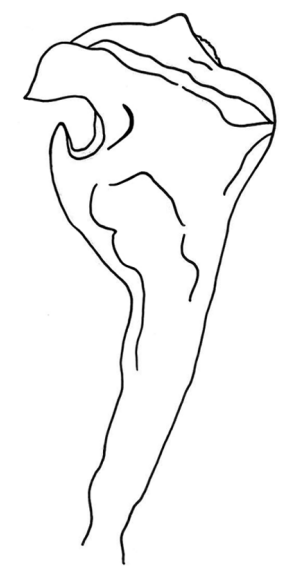

Figura 3. Gonopodo derecho y piezas separadas de gonopodo izquierdo de Hypselodesmus bicolor (topotipo MNHNSD 319.646 ). A-B, gonopodo derecho: A, vista postero-lateral; B, vista antero-lateral. C-D, acropodito izquierdo: C, vista postero-lateral; D, vista antero-lateral. E-F, proceso prefemoral izquierdo: E, vista posterior; F, vista anterior. Escala=1mm. Bordes del proceso prefemoral sombreados en A y B para diferenciar. 
a la depresión alineados. Ausencia de setas dorsales. Ángulo posterior de paraterguito agudo y extendido, sobretodo en segmentos no poríferos, inclinado hacia atrás desde segmentos anteriores, más en los machos que en las hembras. Fórmula porífera normal (5, 7, 9-10, 12-13, 15-19), callo porífero presente, el poro abre lateralmente y hacia arriba. Epiprocto alargado y flexionado en su extremo. Cabeza con surco longitudinal pronunciado a lo largo del vertex. Antenas extendidas hacia atrás sobrepasan margen anterior de S4, más cortas en las hembras. Esternitos frecuentemente más claros que el dorso. Adaptación pregonopodal consistiendo en protuberancia cónica de tamaño apreciable sobre coxas del segundo par de patas. Primeros podómeros gruesos y pilosos, tercero y sexto más largos, patas de las hembras más finas. Hipoprocto ligeramente redondeado, valvas anales estriadas.

Población de Loma Barbacoa: registro de nueva localidad. Machos: longitud $(\mathrm{mm})=29.2$ $(28.0-31.0 ; \mathrm{n}=13)$; anchura $(\mathrm{mm})=4.2(3.9-4.8 ; \mathrm{n}=13)$. Hembras: longitud $(\mathrm{mm})=33.5(31.0-36.0$; $\mathrm{n}=16)$; anchura=4.7 (4.4-5.0; $\mathrm{n}=16)$. MNHNSD के गे19.2,284-2,296; MNHNSD 9 19.2,2972,312. Estos especímenes se encontraron en un manaclar de Loma Barbacoa a 1,227 msnm, simpátricos con H. subtilissimus sp. nov; coordenadas: 358585 E, 2041750 N; colectores: “Chelín”, "Santico", "Naná" y C. Suriel, 18-III-2011 y 01-X-2011. Los tres primeros segmentos presentan el ángulo posterior del paraterguito blanco al igual que los segmentos poríferos, los segmentos no poríferos son castaño oscuro uniforme (marrón oscuro), salvo excepciones en que presentan su ángulo posterior más claro o blanquecino. En algunos segmentos más de la mitad del paraterguito es blanco. La conspicua diferencia de color entre segmentos poríferos y segmentos no poríferos diferencia a la población de Loma Barbacoa (Cordillera Central) de aquellos de la localidad tipo (Loma Quita Espuela, Cordillera Septentrional) y de otros procedentes de la Reserva Científica Ébano Verde (Cordillera Central), además del mayor grosor de los terguitos. Aunque la intensidad y la extensión de los colores suele ser variable en los quelodésmidos de una misma especie, no es común tan notable diferencia en el patrón de color, lo que unido a la separación geográfica de las poblaciones (Cordillera Central-Cordillera Septentrional) hace pensar en la posibilidad de que estemos en presencia de una subespecie.

Ejemplares de Reserva Científica Ébano Verde. Machos: longitud $(\mathrm{mm})=29.0$ (27.5-30.5; $\mathrm{n}=2)$, anchura $(\mathrm{mm})=3.6$; MNHNSD19.2,314-2,315. Hembras $(1)$ : longitud $(\mathrm{mm})=32.0$, anchura $(\mathrm{mm})=3.8$; MNHNSD19.2,316. El Arroyazo: 0338556E-2105988N, 1,160 msnm, bajo musgos, registro de nueva localidad. Características dorsales y ventrales sin diferencias apreciables con respecto a la población tipo.

Topotipos (Reserva Científica Loma Quita Espuela). Dos adultos: MNHNSD đ19.646, longitud $(\mathrm{mm})=29.0$, anchura $(\mathrm{mm})=3.9$; MNHNSD 919.647 , longitud $(\mathrm{mm})=35.0$, anchura $(\mathrm{mm})=4.6$. Colector Antonio R. Pérez-Asso, 10-II-2002. Dos machos juveniles, no numerados; colectores: J. M. Amarante y Carlos Suriel, 2012. En la cima de Loma Quita Espuela, 19²3'N, $70^{\circ} 08^{\prime} \mathrm{O}, 985 \mathrm{msnm}$, bajo musgos, prov. Duarte.

\section{Género Hypselodesmus Loomis, 1941}

Nueva diagnosis. Los gonopodos alcanzan o sobrepasan borde anterior de esternito del séptimo segmento (diferencia respecto al género Podiscodesmus). Acropodito simple, no birramoso (diferencia respecto al género Achromoporus), acintado y curvado, abrazando al proceso prefemoral, su porción distal o postfémur es plegada, estrecha y curvada hacia fuera; estas últimas características lo diferencian de los dos géneros referidos, de Chondrotropis, Beatadesmus y Cyrtaphe. Proceso prefemoral más corto que el acropodito, estrecho en la base, en forma de cinta gruesa en su porción media, retorcido en su porción superior, la que abre con cara excavada dirigida lateralmente o hacia arriba. 
New diagnosis. Gonopods meeting or exceeding anterior margin of sternite of the seventh segment (difference with regard to Podiscodesmus genus). Acropodite simple, with only one branch (difference with regard to Achromoporus genus), band-shaped and bent, around behind the prefemoral process, distally (postfémur) slender, flexure and curving out, these latter characters separate it from the two mentioned genus, and also from Chondrotropis, Beatadesmus and Cyrtaphe. Prefemoral process shorter than the acropodite, narrow at the base, thick bandshaped in its midportion, distally twisted, opening with an excavated face directed laterally or upward.

Nueva descripción. Cuerpo de tamaño mediano, las hembras más grandes que los machos, márgenes laterales casi paralelos, pero los primeros tres segmentos pueden ser ligeramente más anchos.

Vista dorsal. Dorso de color castaño, castaño rojizo o marrón oscuro, pudiendo ser más claro en el centro del margen posterior del metaterguito; paraterguitos, o solamente su ángulo posterior, de un tono ligeramente más claro que el dorso, ambarino o blanco; puede haber diferencia de color entre segmentos poríferos y no poríferos o ser de coloración uniforme. Proterguito y pleurito de tono más oscuro. Ligeramente convexo, sobretodo en segmentos anteriores. Collum subelíptico, ligeramente emarginado en región central del margen posterior, con pequeña depresión anterocentral, más arriba de la porción media del margen anterior, de tamaño mediano o pequeño en relación a la cabeza. Antenas extendidas hacia atrás alcanzan o sobrepasan el margen anterior del tercer segmento. Depresión transversal en centro de metaterguito, tenue o muy marcada, notoria desde el collum o a partir de S3-S5, granos (tubérculos) sutiles o grandes, dispersos, formando una hilera o arreglándose en tres hileras en relación a la depresión, una anterior y dos posteriores, a lo largo del metaterguito y ocupando área de paraterguito. Ausencia de setas. Margen anterior de paraterguito redondeado, margen posterior recto, ligeramente arqueado o inclinado hacia atrás sobrepasando margen posterior del segmento, puede presentar granos. Diente en ángulo anterior de paraterguito a partir de S2, hasta S17-S18 o con expresividad variable, pudiendo faltar o estar solo insinuado en algunos segmentos. El margen lateral del paraterguito puede tener un segundo o un tercer diente. Ligero levantamiento del paraterguito. Ángulo posterior de paraterguito agudo y extendido, con inclinación hacia atrás de expresividad variable, pudiendo ser notoria a partir de segmentos anteriores o medios, pero generalmente en S15-S19, inclinación hacia arriba con expresividad variable. Fórmula porífera normal $(5,7$, 9-10, 12-13, 15-19), poro abriendo lateralmente y hacia arriba; callo porífero presente, pequeño o prominente. Epiprocto alargado con extremo flexionado.

Vista ventral. Surco pronunciado en el vertex de la cabeza, setas en clípeo. Esternitos castaño, frecuentemente más claro que el dorso, con setas. Patas pilosas, tercer y sexto podómeros más largos. Adaptación pregonopodal presente, consistiendo en prominencia cónica sobre las coxas del segundo par de patas.

Gonopodos. Grandes, alcanzando o sobrepasando margen anterior del esternito S7. Prefémur de tamaño mediano o largo. Acropodito simple, de una sola rama, curvado desde su articulación con el prefémur, acintado, más ancho en su base y estrechando progresivamente, abrazando total o parcialmente al proceso prefemoral; postfémur o porción distal delgado, acintado y plegado, curvando hacia fuera, con extremo variable según la especie: bifurcado, agudo o notoriamente filiforme. Un proceso dentiforme en postfémur puede estar presente según la especie. Proceso prefemoral más estrecho en la base, fornido, con porción media como cinta gruesa, parcialmente ocultada por el acropodito; porción superior retorcida, abriendo con una cara ventral excavada que puede mirar hacia arriba o lateralmente, con extremo que se extiende hacia arriba, agudo o irregular; una depresión longitudinal, donde se recuesta el acropodito, puede estar presente de 

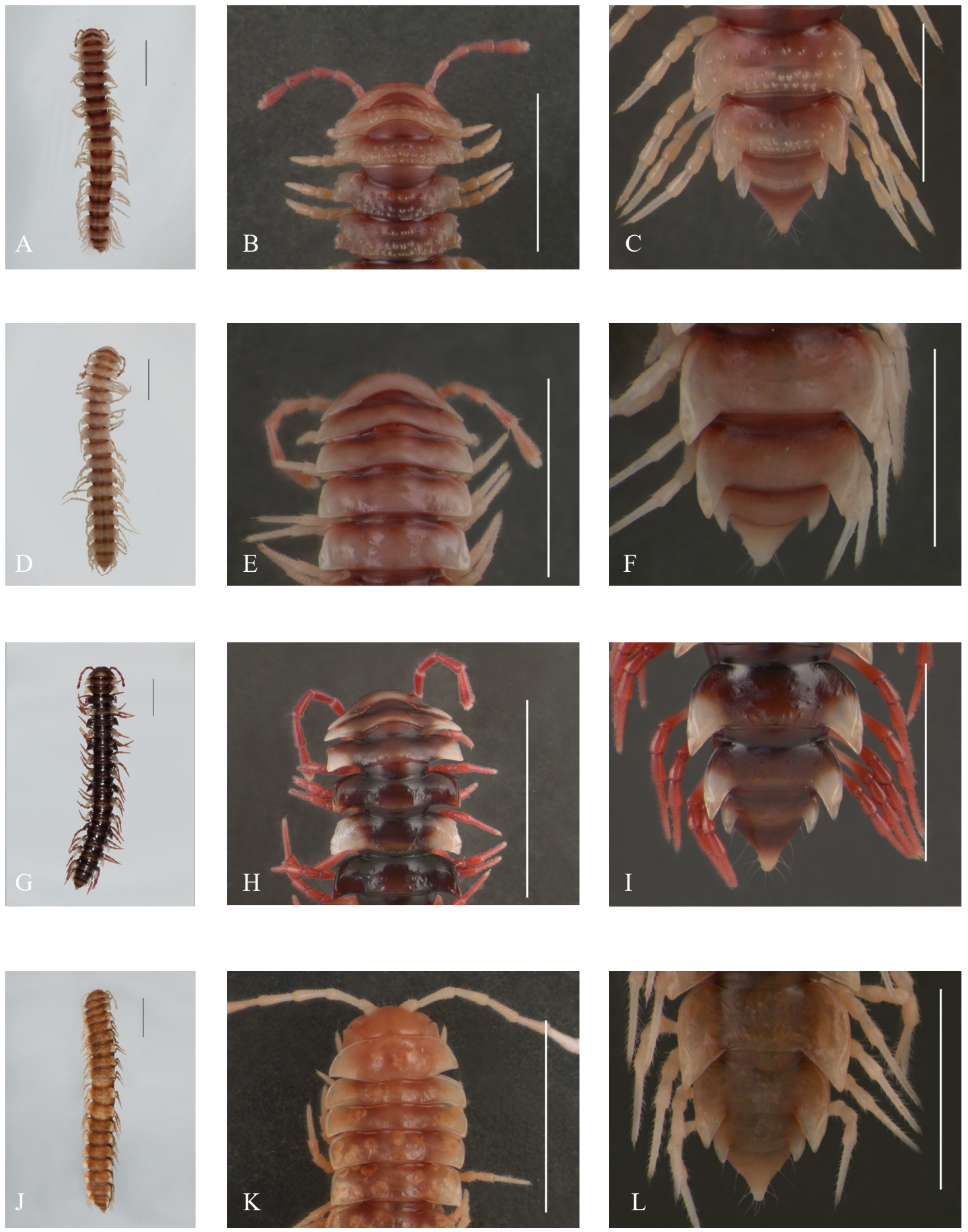

Figura 4. Fotografías de Hypselodesmus subtilissimus sp. nov., Hypselodesmus assoi e Hypselodesmus bicolor (cuerpo enterosegmentos anteriores-segmentos posteriores). A-C, H. subtilissimus sp. nov. (paratipo 19.2,275). D-F, H. assoi (holotipo). G-L, H. bicolor: G-I, ejemplar de Loma Barbacoa; J-L, topotipo de Loma Quita Espuela (ㅇ). Escalas: A,D-L=5mm, B y C=4mm. 
acuerdo a la especie, desde la porción media hasta la parte superior. La cara dorsal de la porción superior puede presentar ligera concavidad o ser convexa de acuerdo a la especie.

Comentario. Los caracteres depresión transversal en metaterguito, granos a lo largo del terguito y dientes paratergales presentan una expresividad progresiva en las tres especies, desde un menor grado en $H$. assoi a uno mayor en $H$. subtilissimus sp. nov., estando $H$. bicolor en posición intermedia.

\section{DISCUSIÓN}

El descubrimiento y descripción de $H$. assoi permitió comenzar a precisar los criterios definitorios de este género en los términos anteriormente descritos (Suriel, 2009). El patrón gonopodal relacionaba sin dudas a la nueva especie con $H$. bicolor, especie tipo del género Hypselodesmus, al tiempo que favorecía iniciar la separación de la variabilidad: prefémur mediano (bicolor) o largo (assoi), acropodito rodeando total (bicolor) o parcialmente (assoi) al proceso prefemoral, extremo del postfémur bifurcado (bicolor) o agudo (assoi), proceso prefemoral abriendo parcialmente en porción superior con cara excavada que mira hacia arriba (bicolor) o abriendo ampliamente con cara excavada que mira lateralmente (assoi). Adicionalmente, otras características no gonopodales comenzaban también a revelar variabilidad: primeros tres segmentos del cuerpo obviamente más anchos (bicolor) o aparentan del mismo ancho, mayor anchura no es muy notoria (assoi); ángulo posterior de paraterguito blanco, más acentuado en segmentos poríferos (bicolor) o solo de un tono más claro que el dorso, no blanco (assoi); diente en ángulo anterior de paraterguito regularmente desde S2 hasta segmentos posteriores (bicolor) o con expresividad muy variable, pudiendo faltar en algunos segmentos o estar apenas insinuado (assoi); igualmente se mostraba en assoi con mucha variabilidad la presencia de la depresión transversal en el centro del metaterguito y los granos asociados a ésta, características registradas en la descripción de bicolor; en el holotipo y en los paratipos de $H$. assoi se mostraba muy tenue la depresión central y los nódulos no se advirtieron, observándose luego en algunos ejemplares de un grupo de topotipos colectados posteriormente.

El nuevo taxon, Hypselodesmus subtilissimus, ha permitido reafirmar la validez del género Hypselodesmus en base al patrón gonopodal ya referido, al tiempo que se aumenta el rango de variabilidad genérica con características específicas muy conspicuas, diferentes a bicolor y assoi: extremo del postfémur notoriamente filiforme (no bifurcado ni agudo), un segundo diente en margen lateral de paraterguito, ocasionalmente un tercer diente (caracteres muy distintivos que le separan de muchas otras especies de la familia), depresión transversal en metaterguito bien marcada (no tenue), profunda en algunos segmentos, con tres hileras de granos o tubérculos grandes, una anterior y dos posteriores respecto a la depresión (no una sola hilera); ángulo posterior de paraterguito inclinado hacia atrás solo en segmentos posteriores (no desde segmentos anteriores o medios).

La descripción de un nuevo taxon, con más razón si es del nivel genérico o más elevado que éste, será siempre una propuesta transitoria, sujeta a precisiones derivadas del descubrimiento y estudio de nuevos morfos y poblaciones, las que revelarán un rango de variabilidad alrededor de uno o varios criterios unitarios y definitorios. Los atributos seleccionados para separar los morfos y definir los nuevos táxones se irán tamizando en la medida que la variabilidad sea conocida.

La taxonomía concebida y practicada de esta manera pasa a ser una disciplina muy dinámica, donde las especies individuales (o los morfos dentro de una misma especie) son las unidades de construcción que permiten ir definiendo los táxones mayores, quedando éstos erigidos "hacia 
arriba", siendo más bien construcciones transitorias que se modificarán de acuerdo aparezcan especies adicionales que obliguen a modificar el concepto de pertenencia que se esté manejando, el que irá quedando definido por el conjunto de las características de todas las especies incluidas (Hoffman, 1979). Este proceder se corresponde, en la taxonomía de los diplópodos, con la tradición establecida por Orator Fuller Cook, al tiempo que niega el "método procusteano" (Hoffman, Ídem), el que se expresa en la incorrecta práctica de establecer estándares arbitrarios fijos a los que deberían ajustarse forzosamente los nuevos morfos descubiertos, siendo excluidos en caso contrario. La consecuencia de este ejercicio artificial de la taxonomía es el establecimiento de numerosas especies, géneros y otros táxones inconsistentes. Se olvida el necesario contenido hipotético-deductivo de la taxonomía, disciplina que debe estar sometida al criterio de falsabilidad y predicción (Fontenla, 2008), como parte de una sistemática que deberá procurar la reconstrucción histórica utilizando diferentes aproximaciones. La propuesta de nuevos táxones asume implícitamente hipótesis evolutivas sobre las relaciones de las especies, las que necesariamente tienen que ser contrastadas con el descubrimiento de nuevos morfos y cruzadas con los resultados obtenidos en otras aproximaciones. El análisis filogenético molecular de los diplópodos de La Hispaniola está pendiente.

En la propuesta de nuevas especies, lo correcto es asumir como criterio de separación, en principio, aquellos rasgos relacionados con las estructuras que suelen ser más conservadoras o que de algún modo estén involucrados con los mecanismos de aislamiento reproductores. En el caso particular de los diplópodos o milpiés, la taxonomía más segura y, en consecuencia, de mayor utilidad es aquella fundamentada en la composición y estructura del aparato de transferencia de esperma del macho o gonopodos. Rasgos tales como: coloración, ornamentaciones dorsales, gradaciones en las formas corporales, dientes paratergales, fórmula porífera, callos poríferos y otros, sin dejar de ser importantes, han resultado ser inestables en muchos casos, presentando en ocasiones apreciable variabilidad entre las especies de un mismo género o entre poblaciones de una misma especie, según el caso. Estudios realizados en las últimas dos décadas con quelodésmidos de Cuba y La Hispaniola son bastante reveladores en este sentido. Pérez-Asso $(1996,1998)$ ha demostrado que las especies del género Amphelictogon Chamberlin, 1918, de Cuba, tanto entre ellas como dentro de cada una, presentan una sorprendente variabilidad en la intensidad, tono y extensión de los colores, así como en otras características externas, siendo el filamentoso postfémur del acropodito, enrrollado en espiral, el único rasgo estable para la separación de las especies, como lo destaca al comparar este caso con el del género Achromoporus Loomis, 1936, de La Hispaniola (Pérez-Asso, 2009).

El caso del género Achromoporus Loomis, 1936, es muy ilustrativo respecto al tema que comentamos. El acropodito birramoso ha sido establecido certeramente como criterio definitorio de este taxon (Pérez-Asso, 2009, 2010), característica consistente que se repite con diferentes grados en $A$. coloratus, $A$. enneryensis, $A$. robustus, A. furcipes y A. heteromus, las cinco especies descritas por Loomis $(1936,1941)$, tomando coloratus como especie tipo. Pero esta consistente propiedad unitaria no fue asumida como tal consecuentemente por el autor del género, razón por la que no la consideró al momento de describir cuatro especies nuevas con el acropodito birramoso, las que, en lugar de incluirlas en Achromoporus, tomó como tipos de sendos géneros nuevos: Lasiomazus, Craterodesmus, Biaporus y Synecheporus (Loomis, 1941). La inadvertencia de un patrón fundamental unificador de estas especies para justificar incluirlas en un mismo género, en gran medida originada por la escasa disponibilidad de ejemplares, creó una situación confusa en la taxonomía dentro de la familia Chelodesmidae en La Hispaniola (Suriel, 2010). Particularmente, nos llamó mucho la atención la descripción de Achromoporus ebanoverde, hecha por Pérez-Asso (2005), especie con acropodito birramoso, pero con una obvia articulación con el prefémur en $90^{\circ}$ como en Lasiomazus Loomis, 1941, al tiempo que mostrando características corporales no propias de este último género. Afortunadamente, como dijéramos más arriba, la situación ha sido resuelta por ese mismo autor en base al examen de 
numerosos ejemplares, topotipos y especies nuevas, proponiendo los cuatro géneros descritos por Loomis en 1941 como sinónimos nuevos de Achromoporus y discerniendo la amplia variabilidad comprendida por el conjunto de las especies (Pérez-Asso, 2009).

La descripción hecha por Loomis de los gonopodos de Hypselodesmus bicolor es fundamentalmente correcta, salvo que la condición "subcilíndrica" del proceso prefemoral (su "inner joint"; Loomis, 1941, p.54) habría que excluirla, toda vez que se trata realmente de una estructura en forma de cinta gruesa, estrecha en su origen, que abre como una lámina retorcida en su porción superior; lo que ocurre es que el acropodito lo oculta, al recostarse sobre la depresión longitudinal que este tiene, de manera que la pieza no se puede apreciar bien en vista posterior de los gonopodos in situ. El extremo bifurcado del acropodito es apreciable en su ilustración de los gonopodos, y muy notorio cuando observamos ejemplares de esta especie al microscopio, pero él no lo menciona en su descripción.

\section{CLAVE DICOTÓMICA PARA LAS ESPECIES DE HYPSELODESMUS}

1-Extremo del postfémur filiforme. Dorso conspicuamente granuloso, con los granos o tubérculos grandes formando tres hileras. H. subtilissimus sp. nov.

-Extremo del postfémur no filiforme. Dorso tenuemente granuloso, granos o tubérculos pequeños sin llegar a formar tres hileras..................................2

2-Extremo del postfémur bifurcado. Ángulo posterior del paraterguito blanco en segmentos poríferos. H. bicolor

-Extremo del postfémur agudo. Ángulo posterior del paraterguito ligeramente más claro que el resto del terguito, pero no blanco, sin diferencia de color entre segmentos poríferos y no poríferos..........H. assoi

\section{AGRADECIMIENTOS}

Agradezco al Fondo Nacional de Innovación y Desarrollo Científico y Tecnológico (FONDOCYT), del Ministerio de Educación Superior, Ciencia y Tecnología (MESCYT), por hacer posible las expediciones a Loma Barbacoa, a través del financiamiento al proyecto "Factores biológicos y geoclimáticos que modelan las comunidades de moluscos y artrópodos terrestres en dos bosques nublados de la República Dominicana" (FONDOCYT No. 2009-102). A Ruth Bastardo, del Instituto de Investigaciones Botánicas y Zoológicas Rafael M. Moscoso (IIBZ-UASD), investigadora principal del referido proyecto, quien también facilitó, junto a Brian Farrell (MCZ, Harvard University), los equipos fotográficos y el programa de procesamiento de fotos del Convenio UASD-Harvard University.

Celeste Mir, Directora del Museo Nacional de Historia Natural (MNHNSD), respaldó las expediciones al Parque Nacional José del Carmen Ramírez y a la Reserva Científica Loma Quita Espuela, también corrigió los textos en inglés del Abstract y las Diagnosis. Antonio R. PérezAsso revisó el manuscrito e hizo importantes correcciones y sugerencias. Gabriel de los Santos (MNHNSD) tomó las fotografías de segmentos anteriores y posteriores de los ejemplares. Luís Díaz (MNHNCu) tomó las fotografías de cuerpo entero. Yurkidia Díaz Féliz (MNHNSD) trabajó la edición de los dibujos con el programa Photoshop CS5. Lon monteros de La Taguía, San Cristóbal, conocidos como "Santico", "Naná" y "Chelín" me sirvieron de guías y asistentes en las expediciones a Loma Barbacoa. A todos ellos extiendo mis agradecimientos. 


\section{LITERATURA CITADA}

Fontenla, J. L. 2008. Taxonmanía. Cocuyo, carta informativa de los zoólogos de invertebrados de las Antillas, 17: 57-68.

Hoffman, R. L. 1979. Clasification of the Diplopoda. Mem. Mus. His. Nat. Geneve. 209 pp.

Hoffman, R. L. 1999. Checklist of the millipedes of North and Middle America. Virg. Mus. Nat. Hist. Spec. Public., 8: 1-584.

Index to organism names (ION), http://www.organismnames.com/query.htm?q., citado en mayo, 2012, Thomson Reuters' Zoological Record.

Jeekel, C. A. W. 1971. Nomenclator generum et familiarum Diplopodorum: A list of the genus and family-group names in the Class Diplopoda from the 10th edition of Linnaeus, 1758, to the end of 1957. Monografieen van de Nederlandse Entomologische Vereniging 5, pp. 1-412 (en línea), http://www.biologie.uniulm.de/systax/, citado mayo 2012.

Loomis, H. F. 1936. The millipeds of Hispaniola, with descriptions of a new family, new genera, and new species. Bull. Mus. Comp. Zool., 80 (1): 3-197.

Loomis, H. F. 1941. Millipeds collected in Puerto Rico and the Dominican Republic by Dr. P. J. Darlington in 1938. Bull. Mus. Comp. Zool., 88 (2): 17-80.

Pérez-Asso, A. R. 1996. Revisión del género Amphelictogon (Diplopoda: Polydesmida: Chelodesmidae) en Cuba. Insecta Mundi, 10: (1-4), 181-216.

Pérez-Asso, A. R. 1998. Nuevas especies del género Amphelictogon (Diplopoda: Polydesmida: Chelodesmidae) en Cuba. Insecta Mundi, 12 (3-4): 161-173.

Pérez-Asso, A. R. 2005. Dos especies y una subespecie nueva de milpiés del género Achromoporus (Diplopoda: Polydesmida) para Hispaniola. Solenodon, 5: 53-59.

Pérez-Asso, A. R. 2009. El género Achromoporus (Diplopoda: Polydesmida: Chelodesmidae) en República Dominicana: especies nuevas y sinonimias. Solenodon, 8: 33-81.

Pérez-Asso, A. R. 2010. Descripción de una especie nueva de milpiés del género Beatadesmus (Diplopoda. Polydesmida: Chelodesmidae) para la República Dominicana: nuevas sinonimias. Novit. Caribaea, 3: 6-12.

Pérez-Asso, A. R. y D. E. Pérez-Gelabert. 2001. Checklist of the millipeds (Diplopoda) of Hispaniola. Boletín S.E.A. 28: 67-80.

Pérez-Gelabert, D. E. 2008. Arthropods of Hispaniola (Dominican Republic and Haiti): A checklist and bibliography. ZOOTAXA 1831: 72.

Suriel, C. 2009. Especie nueva del género Hypselodesmus Loomis (Diplopoda: Polydesmida: Chelodesmidae) del suroeste de la República Dominicana. Novit. Caribaea, 2: 1-6.

Suriel, C. 2010. Dos especies nuevas del género Achromoporus (Diplopoda: Polydesmida: Chelodesmidae) del Parque Nacional José del Carmen Ramírez. Novit. Caribaea, 3: 13-21.

[Recibido: 26 de abril, 2012. Aceptado para publicación: 26 de junio, 2012] 